\title{
Modifications of the Goguen's intuitionistic fuzzy implication
}

\section{Janusz Kacprzyk ${ }^{1}$, Katarína Čunderlíková ${ }^{2}$, Nora Angelova $^{3}$ and Krassimir T. Atanassov ${ }^{4}$}

\author{
${ }^{1}$ Systems Research Institute, Polish Academy of Sciences \\ ul. Newelska 6, 01-447 Warsaw, Poland, and \\ Warsaw School of Information Technology \\ ul. Newelska 6, 01-447 Warsaw, Poland \\ e-mail: kacprzyk@ibspan.waw.pl \\ ${ }^{2}$ Mathematical Institute, Slovak Academy of Sciences \\ Štefánikova 49, 81473 Bratislava, Slovakia \\ e-mail: cunderlikova. lendelova@gmail.com \\ ${ }^{3}$ Faculty of Mathematics and Informatics, Sofia University \\ 5 James Bourchier Blvd., 1164 Sofia, Bulgaria \\ e-mail: nora.angelovaefmi.uni-sofia.bg \\ ${ }^{4}$ Department of Bioinformatics and Mathematical Modelling, \\ Institute of Biophysics and Biomedical Engineering, \\ Bulgarian Academy of Sciences \\ 105 Acad. G. Bonchev Str., 1113 Sofia, Bulgaria \\ e-mail: krat@bas.bg
}

Received: 21 July 2021

Accepted: 20 November 2021

\begin{abstract}
On the basis of the Goguen's intuitionistic fuzzy implication, seven of its modifications are constructed. Some of their basic properties are studied. The negations, generated by these implications are introduced and some of their properties are also described.

Keywords: Intuitionistic fuzzy implication, Intuitionistic fuzzy pair, Goguen's fuzzy implication. 2020 Mathematics Subject Classification: 03 E72.
\end{abstract}




\section{Introduction}

In [6], an intuitionistic fuzzy form of the Joseph Amadee Goguen's (28 June 1941 - 3 July 2006) implication is given and some of its basic properties are studied. Its name is related to the following fuzzy implication (see, e.g., [9]):

$$
a \rightarrow b=\left\{\begin{array}{ll}
1, & \text { if } a \leq b \\
\frac{b}{a}, & \text { otherwise }
\end{array} .\right.
$$

Here, following and combining ideas from [4, 6, 8, 11], we are going to construct 7 new implications, related to the Goguen's intuitionistic fuzzy implication.

\section{Preliminary results}

In the beginning, the necessary concepts from Intuitionistic Fuzzy Pairs theory will be given, following $[5,7]$.

In some definitions we are using functions sg and $\overline{\mathrm{sg}}$ defined by:

$$
\begin{aligned}
& \operatorname{sg}(x)=\left\{\begin{array}{ll}
1 & \text { if } x>0 \\
0 & \text { if } x \leq 0
\end{array},\right. \\
& \overline{\operatorname{sg}}(x)=\left\{\begin{array}{ll}
0 & \text { if } x>0 \\
1 & \text { if } x \leq 0
\end{array} .\right.
\end{aligned}
$$

Let everywhere intuituinistic fuzzy truth values of variables $x$ and $y$ be:

$$
\begin{aligned}
& x=\langle a, b\rangle, \\
& y=\langle c, d\rangle .
\end{aligned}
$$

In [7], these pairs are called Intuituinistic Fuzzy Pairs (IFPs).

The IFP $\langle a, b\rangle$ is:

- a tautology if and only if (iff) $a=1$ and $b=0$;

- an intuitionistic fuzzy tautology (IFT) iff $a \leq c \& b \geq d$.

For two IFPs $x$ and $y$ :

$$
x \leq y \text { iff } a \leq c \text { and } b \geq d .
$$

Let everywhere below we suppose that for each $p>0$ the following condition holds:

$$
\operatorname{sg}(0) \frac{p}{0}=0
$$

The Goguen's intuitionistic fuzzy implications was defined in [6] for the IFPs $x$ and $y$ as:

$$
\langle a, b\rangle \rightarrow\langle c, d\rangle=\left\langle\overline{\operatorname{sg}}(a-c)+\operatorname{sg}(a-c) \frac{c}{1-b}, \operatorname{sg}(a-c) \frac{a-c}{1-b}\right\rangle .
$$


Let us define the empty IFP, the totally uncertain IFP, and the unit IFP (see [7, 5]) by:

$$
\begin{aligned}
& O^{*}=\langle 0,1\rangle, \\
& U^{*}=\langle 0,0\rangle, \\
& E^{*}=\langle 1,0\rangle .
\end{aligned}
$$

Over a fixed IFP $x$ a number of standard and extended modal operators have been defined (see [5]). Here, we use only four of them:

$$
\begin{aligned}
& \square x=\langle a, 1-a\rangle, \\
& \nabla x=\langle 1-b, b\rangle, \\
& \boxplus A=\left\langle\frac{\mu}{2}, \frac{\nu+1}{2}\right\rangle, \\
& \bigotimes A=\left\langle\frac{\mu+1}{2}, \frac{\nu}{2}\right\rangle .
\end{aligned}
$$

\section{Main results}

In [8], if $\supset$ is a fixed implication, the following new modal implication can be introduced:

$$
x \rightarrow y=\square(x \supset y),
$$

where $x$ and $y$ are propositional variables, i.e., having IFP-representation.

Having the Goguen's intuitionistic fuzzy implication $\left(\rightarrow_{G}\right)$ and following [4], we will construct eight new implications, but as we will see below, one of them (the last one) is trivial and it will be omitted.

$$
\begin{aligned}
\langle a, b\rangle \rightarrow_{1}^{*}\langle c, d\rangle & =\square\langle a, b\rangle \rightarrow_{G} \square\langle c, d\rangle \\
& =\left\langle\overline{\operatorname{sg}}(a-c)+\operatorname{sg}(a-c) \frac{c}{a}, \operatorname{sg}(a-c) \frac{a-c}{a}\right\rangle, \\
\langle a, b\rangle \rightarrow_{2}^{*}\langle c, d\rangle & =\square\langle a, b\rangle \rightarrow_{G} \diamond\langle c, d\rangle \\
& =\left\langle\overline{\operatorname{sg}}(a+d-1)+\operatorname{sg}(a+d-1) \frac{1-d}{a}, \operatorname{sg}(a+d-1) \frac{a+d-1}{a}\right\rangle, \\
\langle a, b\rangle \rightarrow_{3}^{*}\langle c, d\rangle & =\diamond\langle a, b\rangle \rightarrow_{G} \diamond\langle c, d\rangle \\
& =\left\langle\overline{\operatorname{sg}}(d-b)+\operatorname{sg}(d-b) \frac{1-d}{1-b}, \operatorname{sg}(d-b) \frac{d-b}{1-b}\right\rangle, \\
\langle a, b\rangle \rightarrow_{4}^{*}\langle c, d\rangle & =\diamond\langle a, b\rangle \rightarrow_{G} \square\langle c, d\rangle \\
& =\left\langle\overline{\operatorname{sg}}(1-b-c)+\operatorname{sg}(1-b-c) \frac{c}{1-b}, \operatorname{sg}(1-b-c) \frac{1-b-c}{1-b}\right\rangle, \\
\langle a, b\rangle \rightarrow_{5}^{*}\langle c, d\rangle & =\boxplus\langle a, b\rangle \rightarrow_{G} \boxplus\langle c, d\rangle \\
& =\left\langle\overline{\operatorname{sg}}(a-c)+\operatorname{sg}(a-c) \frac{c}{1-b}, \operatorname{sg}(a-c) \frac{a-c}{1-b}\right\rangle, \\
\langle a, b\rangle \rightarrow_{6}^{*}\langle c, d\rangle & =\boxplus\langle a, b\rangle \rightarrow_{G} \bigotimes\langle c, d\rangle \\
& =\left\langle\overline{\operatorname{sg}}(a-c)+\operatorname{sg}(a-c) \frac{c+1}{2-b}, \operatorname{sg}(a-c) \frac{a-c}{2-b}\right\rangle,
\end{aligned}
$$




$$
\begin{aligned}
& \langle a, b\rangle \rightarrow_{7}^{*}\langle c, d\rangle=\bigotimes\langle a, b\rangle \rightarrow_{G} \boxplus\langle c, d\rangle=\left\langle\frac{c}{2-b}, \frac{a+1-c}{2-b}\right\rangle, \\
& \langle a, b\rangle \rightarrow_{8}^{*}\langle c, d\rangle=\bigotimes\langle a, b\rangle \rightarrow_{G} \boxplus\langle c, d\rangle=\langle 1,0\rangle .
\end{aligned}
$$

As we mentioned above, the result of implication $\rightarrow_{8}^{*}$ is a constant and by this reason, we eliminate it.

First, we check that the definitions are correct.

For implication $\rightarrow_{1}^{*}$, we obtain.

First, let

$$
\begin{aligned}
X & \equiv \overline{\operatorname{sg}}(a-c)+\operatorname{sg}(a-c) \frac{c}{a}, \\
Y & \equiv \operatorname{sg}(a-c) \frac{a-c}{a}, \\
Z & \equiv X+Y=\overline{\operatorname{sg}}(a-c)+\operatorname{sg}(a-c) \frac{c}{a}+\operatorname{sg}(a-c) \frac{a-c}{a} .
\end{aligned}
$$

If $a>c$, then $\operatorname{sg}(a-c)=1, \overline{\operatorname{sg}}(a-c)=0$ and hence

$$
\begin{aligned}
0 \leq X & =0+\frac{c}{a}<1, \\
Y & =\frac{a-c}{a}, \\
Z & =\frac{c}{a}+\frac{a-c}{a}=1 .
\end{aligned}
$$

If $a \leq c$, then $\operatorname{sg}(a-c)=0, \overline{\operatorname{sg}}(a-c)=1$ and hence

$$
\begin{aligned}
& X=1, \\
& Y=0, \\
& Z=1,
\end{aligned}
$$

i.e., the implication $\rightarrow_{1}^{*}$ is correct. By the same manner, we check of the correctness of the rest implications.

Second, for the basic properties of implications we obtain that:

$$
\begin{aligned}
& O^{*} \rightarrow_{i}^{*} O^{*} \text { for } i=1,2,3,4,5,6, \\
& O^{*} \rightarrow_{i}^{*} U^{*} \text { for } i=1,2,3,4,5,6, \\
& O^{*} \rightarrow_{i}^{*} E^{*} \text { for } i=1,2,3,4,5,6,7, \\
& U^{*} \rightarrow_{i}^{*} O^{*} \text { for } i=1,2,5,6, \\
& U^{*} \rightarrow_{i}^{*} U^{*} \text { for } i=1,2,3,5,6, \\
& U^{*} \rightarrow_{i}^{*} E^{*} \text { for } i=1,2,3,4,5,6,7, \\
& E^{*} \rightarrow{ }_{i}^{*} O^{*} \text { for } i=6, \\
& E^{*} \rightarrow{ }_{i}^{*} U^{*} \text { for } i=2,3,6, \\
& E^{*} \rightarrow{ }_{i}^{*} E^{*} \text { for } i=1,2,3,4,5,6,7
\end{aligned}
$$


are IFTs and

$$
\begin{aligned}
& O^{*} \rightarrow_{i}^{*} O^{*} \text { for } i=1,2,3,4,5,6, \\
& O^{*} \rightarrow_{i}^{*} U^{*} \text { for } i=1,2,3,4,5,6, \\
& O^{*} \rightarrow_{i}^{*} E^{*} \text { for } i=1,2,3,4,5,6,7, \\
& U^{*} \rightarrow_{i}^{*} O^{*} \text { for } i=1,2,5,6, \\
& U^{*} \rightarrow_{i}^{*} U^{*} \text { for } i=1,2,3,5,6, \\
& U^{*} \rightarrow_{i}^{*} E^{*} \text { for } i=1,2,3,4,5,6, \\
& E^{*} \rightarrow{ }_{i}^{*} U^{*} \text { for } i=2,3, \\
& E^{*} \rightarrow{ }_{i}^{*} E^{*} \text { for } i=1,2,3,4,5,6
\end{aligned}
$$

are tautologies.

Third, let us mention by $\supset_{i}$ the expression $x \rightarrow_{i} y$. Then we formulate and prove the next theorem.

Theorem 1. The following relations hold:

$$
\supset_{2} \geq\left\{\begin{array}{l}
\supset_{1} \geq \supset_{5} \\
\supset_{3} \\
\supset_{6}
\end{array}\right\} \geq \supset_{4} \geq \supset_{7} .
$$

Proof. For the check that $\supset_{2} \geq \supset_{1}$, we put:

$$
X \equiv \overline{\operatorname{sg}}(a+d-1)+\operatorname{sg}(a+d-1) \frac{1-d}{a}-\overline{\operatorname{sg}}(a-c)+\operatorname{sg}(a-c) \frac{c}{a}
$$

and

$$
Y \equiv \operatorname{sg}(a-c) \frac{a-c}{a}-\operatorname{sg}(a+d-1) \frac{a+d-1}{a} .
$$

If $a>1-d(\geq c)$, then

$$
X=0+1 \cdot \frac{1-d}{a}-0+1 \cdot \frac{c}{a}=\frac{1-d}{a}-\frac{c}{a}=\frac{1-c-d}{a} \geq 0
$$

and

$$
Y=1 \cdot \frac{a-c}{a}-1 \cdot \frac{a+d-1}{a}=\frac{1-c-d}{a} \geq 0 .
$$

If $1-d \geq a$, then

$$
\begin{gathered}
X=1+0 \cdot \frac{1-d}{a}-\overline{\operatorname{sg}}(a-c)+\operatorname{sg}(a-c) \frac{c}{a}=1-\overline{\operatorname{sg}}(a-c)+\operatorname{sg}(a-c) \frac{c}{a} \\
\geq \operatorname{sg}(a-c) \frac{c}{a} \geq 0 .
\end{gathered}
$$

and

$$
Y=\operatorname{sg}(a-c) \frac{a-c}{a}-0 . \frac{a+d-1}{a} \geq 0 .
$$

All other relations are proved in the same manner. 
Fourth, following $[5,7,10]$, we define the classical intuitionistic fuzzy negation by:

$$
\begin{aligned}
\neg_{1}^{*} x & =x \rightarrow{ }_{1}^{*} O^{*}=\langle\overline{\operatorname{sg}}(a), \operatorname{sg}(a)\rangle, \\
\neg_{2}^{*} x & =x \rightarrow{ }_{2}^{*} O^{*}=\langle\overline{\operatorname{sg}}(a), \operatorname{sg}(a)\rangle, \\
\neg_{3}^{*} x & =x \rightarrow \rightarrow_{3}^{*} O^{*}=\langle\overline{\operatorname{sg}}(1-b), \operatorname{sg}(1-b)\rangle, \\
\neg_{4}^{*} x & =x \rightarrow \rightarrow_{4}^{*} O^{*}=\langle\overline{\operatorname{sg}}(1-b), \operatorname{sg}(1-b)\rangle, \\
\neg_{5}^{*} x & =x \rightarrow \rightarrow_{5}^{*} O^{*}=\left\langle\overline{\operatorname{sg}}(a), \operatorname{sg}(a) \frac{a}{1-b}\right\rangle=\left\langle\overline{\operatorname{sg}}(a), \frac{a}{1-b}\right\rangle, \\
\neg_{6}^{*} x & =x \rightarrow \rightarrow_{6}^{*} O^{*}=\left\langle\overline{\operatorname{sg}}(a)+\operatorname{sg}(a) \frac{1}{2-b}, \operatorname{sg}(a) \frac{a}{2-b}\right\rangle=\left\langle\overline{\operatorname{sg}}(a)+\frac{\operatorname{sg}(a)}{2-b}, \frac{a}{2-b}\right\rangle, \\
\neg_{7}^{*} x & =x \rightarrow{ }_{7}^{*} O^{*}=\left\langle 0, \frac{a+1}{2-b}\right\rangle,
\end{aligned}
$$

because obviously, for each $a \in[0,1]: a \cdot \operatorname{sg}(a)=a$.

We see that

$$
\begin{aligned}
& \neg_{1}^{*} x=\neg_{2}^{*} x=\neg_{2} x, \\
& \neg_{3}^{*} x=\neg_{4}^{*} x=\neg_{5} x,
\end{aligned}
$$

where $\neg_{2}$ and $\neg_{5}$ are negations, described in details in [5], while the negations $\neg_{5}^{*}, \neg_{6}^{*}$, and $\neg_{7}^{*}$ are new ones and in near future will obtain their specific numerations.

For the new negations we check:

$$
\neg_{5}^{*} \neg_{5}^{*} x=\neg_{5}^{*}\left\langle\overline{\operatorname{sg}}(a), \frac{a}{1-b}\right\rangle=\left\langle\operatorname{sg}(a), \frac{\overline{\operatorname{sg}}(a)(1-b)}{1-a-b}\right\rangle,
$$

because $\overline{\operatorname{sg}}(\overline{\operatorname{sg}}(a))=\operatorname{sg}(a)$. Therefore,

$$
\neg_{5}^{*} \neg_{5}^{*} x \neq x
$$

i.e., this negation is not a classical negation. For it we check also:

$$
\neg_{5}^{*} \neg_{5}^{*} \neg_{5}^{*} x=\neg_{5}^{*}\left\langle\operatorname{sg}(a), \frac{\overline{\operatorname{sg}}(a)(1-b)}{1-a-b}\right\rangle=\left\langle\overline{\operatorname{sg}}(a), \frac{\operatorname{sg}(a)(1-a-b)}{1-a-b-\overline{\operatorname{sg}}(a)(1-b)}\right\rangle,
$$

because $\overline{\operatorname{sg}}(\operatorname{sg}(a))=\overline{\operatorname{sg}}(a)$.

Now, let

$$
X \equiv \frac{\operatorname{sg}(a)(1-a-b)}{1-a-b-\overline{\operatorname{sg}}(a)(1-b)}-\frac{a}{1-b} .
$$

If $a=0$, then by the above condition $(*)$ :

$$
X=0 . \frac{1-b}{0}-\frac{0}{1-b}=0 .
$$

If $a>0$, then

$$
X=\frac{1-a-b}{1-a-b}-\frac{a}{1-b}=1-\frac{a}{1-b}=-\frac{1-a-b}{1-b} \geq 0 .
$$

Therefore,

$$
\neg_{5} x \geq \neg_{5}^{*} \neg_{5}^{*} \neg_{5}^{*} x,
$$

i.e., not always the well-known logical equality $\neg x=\neg \neg \neg x$ (see, e.g., $[8,10]$ ) is true. 
Also, if

$$
\begin{aligned}
x \rightarrow_{5}^{*} \neg_{5}^{*} \neg_{5}^{*} x & =\langle a, b\rangle \rightarrow_{5}^{*}\left\langle\operatorname{sg}(a), \frac{\overline{\operatorname{sg}}(a)(1-b)}{1-a-b}\right\rangle \\
& =\left\langle\overline{\operatorname{sg}}(a-\operatorname{sg}(a))+\operatorname{sg}(a-\operatorname{sg}(a)) \frac{\operatorname{sg}(a)}{1-b}, \operatorname{sg}(a-\operatorname{sg}(a)) \frac{a-\operatorname{sg}(a)}{1-b}\right\rangle \\
& =\langle 1,0\rangle,
\end{aligned}
$$

because if $a=0$, then $\overline{\operatorname{sg}}(a-\operatorname{sg}(a))=\overline{\operatorname{sg}}(0)=1$ and $\operatorname{sg}(a-\operatorname{sg}(a))=\operatorname{sg}(0)=0$; and if $a>0$, then $\overline{\operatorname{sg}}(a-\operatorname{sg}(a))=\overline{\operatorname{sg}}(a-1)=1$ and $\operatorname{sg}(a-\operatorname{sg}(a))=\operatorname{sg}(a-1)=0$.

Therefore, the intuitionistic condition $x \rightarrow \neg \neg x$ is satisfied as an IFT, as well as, as a tautology.

On the other hand,

$$
\begin{aligned}
\neg_{5}^{*} \neg_{5}^{*} x \rightarrow_{5}^{*} x & =\left\langle\operatorname{sg}(a), \frac{\overline{\operatorname{sg}}(a)(1-b)}{1-a-b}\right\rangle \rightarrow_{5}^{*}\langle a, b\rangle \\
& =\left\langle\overline{\operatorname{sg}}(\operatorname{sg}(a)-a)+\operatorname{sg}(\operatorname{sg}(a)-a) \frac{a}{1-\frac{\overline{\operatorname{sg}}(a)(1-b)}{1-a-b}}, \operatorname{sg}(\operatorname{sg}(a)-a) \frac{\operatorname{sg}(a)-a}{1-\frac{\overline{\operatorname{sg}}(a)(1-b)}{1-a-b}}\right\rangle
\end{aligned}
$$

and it is not a tautology, because, e.g., for $0<a<1$

$$
\begin{aligned}
\neg_{5}^{*} \neg_{5}^{*} x \rightarrow_{5}^{*} x & =\left\langle\overline{\operatorname{sg}}(1-a)+\operatorname{sg}(1-a) \frac{a}{1-\frac{0}{1-a-b}}, \operatorname{sg}(1-a) \frac{1-a}{1-\frac{0}{1-a-b}}\right\rangle \\
& =\langle\overline{\operatorname{sg}}(1-a)+\operatorname{sg}(1-a) \cdot a, \operatorname{sg}(1-a) \cdot(1-a)\rangle \\
& =\langle a, 1-a\rangle
\end{aligned}
$$

that is not an IFT when $a<0.5$ and therefore, it is not a tautology. By the same manner we can check the relations for $\rightarrow_{6}$ and $\rightarrow_{7}$ and can formulate the following theorem.

Theorem 2. For each IFP $x$,

a) $x \rightarrow \neg \neg x$ is a tautology for $\rightarrow \rightarrow_{5}^{*}$ and $\neg_{5}^{*}$, but not for $\rightarrow_{6}^{*}$ and $\neg_{6}^{*}$, and for $\rightarrow_{7}^{*}$ and $\neg{ }_{7}^{*}$;

b) $x \rightarrow \neg \neg x$ is an IFT for $\rightarrow_{5}^{*}$ and $\neg_{5}^{*}$, and for $\rightarrow_{6}^{*}$ and $\neg_{6}^{*}$, but not for $\rightarrow{ }_{7}^{*}$ and $\neg_{7}^{*}$;

c) $\neg \neg x \rightarrow x$ is not a tautology for the new implications and negations;

d) $\neg \neg x \rightarrow x$ is an IFT for $\rightarrow \rightarrow_{6}^{*}$ and $\neg_{6}^{*}$, but not for $\rightarrow_{5}^{*}$ and $\neg_{5}^{*}$, and for $\rightarrow{ }_{7}^{*}$ and $\neg_{7}^{*}$;

e) $\neg \neg \neg x=\neg x$ is not a tautology for the new implications and negations;

f) $\neg \neg \neg x=\neg x$ is not an IFT for the new implications and negations.

Fifth, we mention that nine axioms for implications are introduced in [9]. They are the following:

- Axiom A1 $(\forall x, y)(x \leq y \rightarrow(\forall z)(I(x, z) \geq I(y, z)))$.

- Axiom A2 $(\forall x, y)(x \leq y \rightarrow(\forall z)(I(z, x) \leq I(z, y)))$. 
- Axiom A3 $(\forall y)\left(I\left(0^{*}, y\right)=E^{*}\right)$.

- Axiom A4 $(\forall y)\left(I\left(E^{*}, y\right)=y\right)$.

- Axiom A5 $(\forall x)\left(I(x, x)=E^{*}\right)$.

- Axiom $\mathbf{A 6}(\forall x, y, z)(I(x, I(y, z))=I(y, I(x, z)))$.

- Axiom $\mathbf{A 7}(\forall x, y)\left(I(x, y)=E^{*}\right.$ iff $\left.x \leq y\right)$.

- Axiom $\mathbf{A 8}(\forall x, y)(I(x, y)=I(N(y), N(x)))$, where $N$ is an operation for a negation.

- Axiom A9 $I$ is a continuous function.

Following [5], we will mention that if some axiom is valid as an IFT, the number of the axiom is marked with an asterisk $\left(^{*}\right)$. These axioms are:

- Axiom A3* $(\forall y)(I(0, y)$ is an IFT).

- Axiom A4* $(\forall y)(I(1, y) \leq y)$

- Axiom A5* $(\forall x)(I(x, x)$ is an IFT).

- Axiom A7* $(\forall x, y)(I(x, y)$ is an IFT iff $x \leq y)$.

- Axiom A8* $(\forall x, y)(I(x, y)=N(N(I(N(y), N(x)))))$.

The following two theorems are proved by the manner, used in the proof of Theorem 1 .

Theorem 3. The following implications satisfy the following axioms as tautologies:

A1: $\rightarrow_{1}^{*}, \rightarrow_{2}^{*}, \rightarrow_{3}^{*}, \rightarrow_{4}^{*}$

A2: $\rightarrow_{1}^{*}, \rightarrow_{2}^{*}, \rightarrow_{3}^{*}, \rightarrow_{4}^{*}, \rightarrow_{5}^{*}, \rightarrow_{6}^{*}$;

A3: $\rightarrow{ }_{1}^{*}, \rightarrow_{2}^{*}, \rightarrow_{3}^{*}, \rightarrow_{4}^{*}, \rightarrow_{5}^{*}, \rightarrow_{6}^{*}$;

$\mathbf{A} 3^{*}: \rightarrow_{1}^{*}, \rightarrow_{2}^{*}, \rightarrow_{3}^{*}, \rightarrow_{4}^{*}, \rightarrow_{5}^{*}, \rightarrow_{6}^{*}$

A4: no one;

A4*: $\rightarrow_{1}^{*}, \rightarrow_{4}^{*}, \rightarrow_{5}^{*}, \rightarrow_{7}^{*}$

A5: $\rightarrow{ }_{1}^{*}, \rightarrow_{2}^{*}, \rightarrow_{3}^{*}, \rightarrow_{5}^{*}, \rightarrow_{6}^{*}$;

$\mathbf{A 5}^{*}: \rightarrow_{1}^{*}, \rightarrow_{2}^{*}, \rightarrow_{3}^{*}, \rightarrow_{5}^{*}, \rightarrow_{6}^{*}$;

A6: $\rightarrow_{1}^{*}, \rightarrow_{2}^{*}, \rightarrow_{3}^{*}, \rightarrow_{4}^{*}$;

A7: no one;

A7*: no one;

A8: no one;

A8*: no one;

A9: if condition $(*)$ is true, all implications. 
Theorem 4. The implications satisfy the following axioms as IFTs:

A1: $\rightarrow_{1}^{*}, \rightarrow_{2}^{*}, \rightarrow_{3}^{*}, \rightarrow_{4}^{*}, \rightarrow_{6}^{*}$;

A2: $\rightarrow_{1}^{*}, \rightarrow_{2}^{*}, \rightarrow_{3}^{*}, \rightarrow_{4}^{*}, \rightarrow_{5}^{*}, \rightarrow_{6}^{*}$

A3: $\rightarrow_{1}^{*}, \rightarrow_{2}^{*}, \rightarrow_{3}^{*}, \rightarrow_{4}^{*}, \rightarrow_{5}^{*}, \rightarrow_{6}^{*}$

$\mathbf{A} 3^{*}: \rightarrow_{1}^{*}, \rightarrow_{2}^{*}, \rightarrow_{3}^{*}, \rightarrow_{4}^{*}, \rightarrow_{5}^{*}, \rightarrow_{6}^{*}$

A4: no one;

$\mathbf{A} 4 *: \rightarrow_{1}^{*}, \rightarrow_{4}^{*}, \rightarrow_{5}^{*}, \rightarrow_{7}^{*}$

A5: $\rightarrow_{1}^{*}, \rightarrow_{2}^{*}, \rightarrow_{3}^{*}, \rightarrow_{5}^{*}, \rightarrow_{6}^{*}$

$\mathbf{A 5 *}: \rightarrow_{1}^{*}, \rightarrow_{2}^{*}, \rightarrow_{3}^{*}, \rightarrow_{5}^{*}, \rightarrow_{6}^{*}$;

A6: $\rightarrow_{1}^{*}, \rightarrow_{2}^{*}, \rightarrow_{3}^{*}, \rightarrow_{4}^{*}$;

A7: no one;

A7*: no one;

A8: no one;

A8*: no one;

A9: if condition (*) is true, all implications.

Following the notation from [5], in future we will denote implications $\rightarrow_{1}^{*}, \ldots, \rightarrow_{7}^{*}$ as $\rightarrow_{199}$ $, \ldots, \rightarrow_{205}$ and we will denote negations $\neg_{5}^{*}, \neg_{6}^{*}$ and $\neg_{7}^{*}$ as $\neg_{53}, \neg_{54}, \neg_{55}$.

\section{Conclusion}

Following [6], we mention that as it is shown in [1, 2, 3], for each intuitionistic fuzzy implication, one or three intuitionistic fuzzy disjunctions and conjunctions are introduced. For example, for disjunction, the following formulas are used:

$$
\begin{aligned}
& p \vee_{1} q=\neg p \rightarrow q, \\
& p \vee_{2} q=\neg p \rightarrow \neg \neg q,
\end{aligned}
$$

where operation intuitionistic fuzzy negation $(\neg)$ is generated by the respective intuitionistic fuzzy implication $(\rightarrow)$; and

$$
p \vee_{3} q=\neg p \rightarrow q,
$$

where the negation $\neg$ is the classical one.

In future, the re pective intuitionistic fuzzy disjunctions and conjunctions, associated with the seven new implications will be introduced and their properties will be studied. Having in mind the form of the negations, generated by the new implications, we must immediately mention that each of these implications will generate three different disjunctions and conjunctions. 


\section{Acknowledgements}

The first and the fourth authors are grateful grateful for the support provided under the joint research project of the Bulgarian Academy of Sciences and the Polish Academy of Sciences, entitled "Intuitionistic Fuzzy Sets - Theory and Applications in Medicine, Economy and other areas".

The second and the fourth authors are grateful for the support provided under the joint research project of the Bulgarian Academy of Sciences and the Slovak Academy of Sciences, entitled "Generation and Applications of Probabilistic and Intuitionistic Fuzzy Models of Uncertainty".

\section{References}

[1] Angelova, N., \& Stoenchev, M. (2015/2016). Intuitionistic fuzzy conjunctions and disjunctions from first type. Annual of "Informatics" Section, Union of Scientists in Bulgaria, 8, 1-17.

[2] Angelova, N., Stoenchev, M., \& Todorov, V. (2017). Intuitionistic fuzzy conjunctions and disjunctions from second type. Issues in Intuitionistic Fuzzy Sets and Generalized Nets, 13, $143-170$.

[3] Angelova, N., \& Stoenchev, M. (2017). Intuitionistic fuzzy conjunctions and disjunctions from third type. Notes on Intuitionistic Fuzzy Sets, 23(5), 29-41.

[4] Atanassov, K. (2006). A new intuitionistic fuzzy implication from a modal type. Advanced Studies on Contemporary Mathematics, 12(1), 117-122.

[5] Atanassov, K. (2017). Intuitionistic Fuzzy Logics, Springer, Cham.

[6] Atanassov, K., Angelova, N., \& Atanassova, V. (2021). On an Intuitionistic Fuzzy Form of the Goguen's Implication. Mathematics, 9(6), Article No. 676.

[7] Atanassov, K., Szmidt, E., \& Kacprzyk, J. (2013). On intuitionistic fuzzy pairs. Notes on Intuitionistic Fuzzy Sets, 19(3), 1-13.

[8] Feys, R. (1965). Modal Logics. Gauthier-Villars, Paris.

[9] Klir, G., \& Yuan, B. (1995). Fuzzy Sets and Fuzzy Logic. Prentice Hall, New Jersey.

[10] Mendelson, E. (1964). Introduction to Mathematical Logic, Princeton, NJ: D. Van Nostrand.

[11] Michalíková, A., Szmidt, E., \& Vassilev, P. (2021). Modifications of Lukasiewicz's intuitionistic fuzzy implication. Notes on Intuitionistic Fuzzy Sets, 27(3), 32-39. 